OPEN ACCESS

Edited by:

Louis Bherer,

Université de Montréal, Canada

Reviewed by:

Arun Bokde,

Trinity College, Dublin, Ireland

Betty M. Tijms,

VU University Medical Center, Netherlands

Teresa Liu-Ambrose,

University of British Columbia,

Canada

*Correspondence:

Yin Wu

wuyin@sus.edu.cn

${ }^{\dagger}$ These authors have contributed equally to this work.

Received: 10 July 2016 Accepted: 12 December 2016 Published: 04 January 2017

Citation:

Tan X-Y, Pi Y-L, Wang J, Li X-P, Zhang L-L, Dai W, Zhu H, Ni Z, Zhang $J$ and $W u Y$ (2017) Morphological and Functional Differences between Athletes and

Novices in Cortical

Neuronal Networks.

Front. Hum. Neurosci. 10:660. doi: 10.3389/fnhum.2016.00660

\section{Morphological and Functional Differences between Athletes and Novices in Cortical Neuronal Networks}

\author{
Xiao-Ying Tan ${ }^{1 \dagger}$, Yan-Ling $\mathrm{Pi}^{2 \dagger}$, Jue Wang ${ }^{3}$, Xue-Pei Li ${ }^{4}$, Lan-Lan Zhang ${ }^{4}$, Wen Dai ${ }^{4}$, \\ Hua Zhu ${ }^{4}$, Zhen $\mathrm{Ni}^{5}$, Jian Zhang ${ }^{4}$ and $\mathrm{Yin} \mathrm{Wu}^{6 *}$ \\ ${ }^{1}$ School of Physical Education and Coaching, Shanghai University of Sport, Shanghai, China, ${ }^{2}$ Shanghai Punan Hospital \\ of Pudong New District, Shanghai, China, ${ }^{3}$ Institutes of Psychological Sciences, HangZhou Normal University, Hangzhou, \\ China, ${ }^{4}$ School of Kinesiology, Shanghai University of Sport, Shanghai, China, ${ }^{5}$ Division of Neurology, Krembil Neuroscience \\ Centre and Toronto Western Research Institute, University Health Network, University of Toronto, Toronto, ON, Canada, \\ ${ }^{6}$ School of Economics and Management, Shanghai University of Sport, Shanghai, China
}

The cortical structural and functional differences in athletes and novices were investigated with a cross-sectional paradigm. We measured the gray matter volumes and resting-state functional connectivity in 21 basketball players and 21 novices with magnetic resonance imaging (MRI) techniques. It was found that gray matter volume in the left anterior insula (Al), inferior frontal gyrus (IFG), inferior parietal lobule (IPL) and right anterior cingulate cortex (ACC), precuneus is greater in basketball players than that in novices. These five brain regions were selected as the seed regions for testing the resting-state functional connectivity in the second experiment. We found higher functional connectivity in default mode network, salience network and executive control network in basketball players compared to novices. We conclude that the morphology and functional connectivity in cortical neuronal networks in athletes and novices are different.

\begin{abstract}
Keywords: basketball player, motor expertise, magnetic resonance imaging, plasticity, resting state functional connectivity
\end{abstract}

\section{INTRODUCTION}

Cortical plasticity is an intrinsic property of the human brain and occurs after long-term training under various conditions (Blakemore and Frith, 2005; Pascual-Leone et al., 2005). Structural differences were found in regional cortical morphology between musicians and non-musicians (Gaser and Schlaug, 2003). Interestingly, London taxi drivers have larger gray matter volume than that in healthy controls or even non-taxi drivers in posterior hippocampi where information of spatial representation is stored (Maguire et al., 2000). However, it is not clear whether long-term training may have effects on cortical morphology with plasticity in motor related cortical areas and whether these effects may contribute to the improvement in motor functions. Elite athletes in the confrontational sports (e.g., basketball etc.) start training since childhood. The sophisticated skills in confrontational sports are likely due to the involvement of different brain areas related to various cortical networks (di Pellegrino et al., 1992; Gallese et al., 1996). These elite athletes offer a special model for studying the long-term training related cortical plasticity with changes in multiple brain areas. In contrast to the long time period required for the longitudinal studies (or often nearly impossible due to extremely long time consumption), a cross-sectional 
paradigm (cohort paradigm) with a comparison between highly skilled elite athletes and novices was widely used recently (Imfeld et al., 2009; Jäncke et al., 2009; Wei et al., 2011; Fauvel et al., 2014). Although the cross-sectional paradigm may not be more sufficient than the longitudinal paradigm, the study using cross-sectional paradigm is more practicable and the results obtained from a cross-sectional designed study also provide an important indication of the presence of plasticity effects with long-term training. Voxel-based morphometry (VBM) is a neuroimaging analysis technique that allows investigation of focal differences in brain anatomy (Ashburner and Friston, 2000). In the present study, we compared the gray matter volumes in various brain areas in basketball players with those in novices in a cross-sectional paradigm by measuring the structural variation in cortical morphology with VBM. We hypothesize that the gray matter volumes in motor related cortical areas are different between basketball players and novices. In addition, since long-term training process leads to refined cognitive functions such as visual search (Williams et al., 1999; McRobert et al., 2007) and sensory perceptions (Aglioti et al., 2008; Wu et al., 2013) in elite athletes, we also expect that the morphological difference between basketball player and novices may be present in cortical areas responsible for cognitive functions.

Motor expertise involves several internal processes requiring organization and integration of sensory and motor information in different cortical areas (Lisberger, 1988). Neuroimaging studies have showed that the human brain is intrinsically organized into a set of spatially distributed, functionally specific networks (Damoiseaux et al., 2006; Bressler and Menon, 2010). Cortical plasticity with long-term training to gain motor expertise is complex. The interaction of cortical activation among different brain areas at the network level may be associated with multi-factorial process of cortical plasticity (Dosenbach et al., 2008). In particular, default mode network, salience network and executive control network are major functional networks relevant to motor and cognitive functions (Bressler and Menon, 2010; Cocchi et al., 2014). Default mode network is activated during motor related spontaneous cognition (Buckner et al., 2008; Mantini and Vanduffel, 2013). The salience network plays an important role in guiding orientation of attention and monitoring of errors during events with internal and external activities (Eckert et al., 2009). The executive control network is responsible for high-level cognitive functions during motor behaviors (Alvarez and Emory, 2006; Fox et al., 2006). Our second hypothesis is that the resting-state functional connectivity in basketball players and novices are different, as relatively less evidence was found in functional brain network in top athletes. We selected the cortical areas with larger gray matter volumes in basketball players (compared to novices, defined in the VBM analysis) as the seed regions and used a seed-based approach to test the functional connectivity in two subject groups. We predicted that the different resting-state functional connectivity between basketball players and novices will be related to the cortical areas located in default mode network, salience network and executive control network which are relevant to the motor and cognitive functions in the basketball players.

\section{MATERIALS AND METHODS}

\section{Participants}

Twenty-one basketball players (mean age $21.3 \pm 1.3$ years, age range 18-23 years) and 21 novices (mean age $21.9 \pm 0.8$ years, age range 19-24 years) were studied. All subjects were male (Shanghai University of Sport is one of the major training centers for men's basketball in China). The basketball players were national first-class athletes and were trained five sessions per week (each daily session about $3 \mathrm{~h}$ ) for 10-15 years (mean duration, $11.4 \pm 2.3$ years). The novices were university students without professional training in basketball or any other sports. Basketball players were taller $(190.6 \pm 3.4 \mathrm{~cm})$ than healthy controls $(176.8 \pm 2.9 \mathrm{~cm} ; t=14.1, p<0.001)$. The experimental protocol was approved by the regional ethics committee of the Shanghai University of Sport. All subjects gave written informed consent in accordance with the Declaration of Helsinki.

\section{Magnetic Resonance Imaging}

Imaging scanning was performed using a 3T Siemens scanner in the functional magnetic resonance imaging (fMRI) center at the East China Normal University. The anatomical difference in gray matter volume between basketball players and novices was tested using the T1-weighted structural image scanning. A high-resolution image with 192 slices was acquired using a 3-dimension fast-field echo sequence (echo time $(\mathrm{TE})=2.34 \mathrm{~ms}$, repetition time $(\mathrm{TR})=2530 \mathrm{~ms}$, flip angle $(\mathrm{FA})=7^{\circ}$, field of view $(\mathrm{FOV})=256 \mathrm{~mm}^{2} \times 256 \mathrm{~mm}^{2}$, slice thickness $=1 \mathrm{~mm}$, inversion time $(\mathrm{TI})=1100 \mathrm{~ms})$. The restingstate $\mathrm{fMRI}$ scanning was performed with a gradient echo planar imaging sequence $\left(\mathrm{TE}=30 \mathrm{~ms}, \mathrm{TR}=2000 \mathrm{~ms}, \mathrm{FA}=90^{\circ}\right.$, FOV $=240 \mathrm{~mm}^{2} \times 240 \mathrm{~mm}^{2}$, slice thickness $\left.=3 \mathrm{~mm}\right)$. A total of 210 scans were obtained from each subject. Subjects were instructed to keep themselves relaxed while lying still in the scanner with their eyes closed.

\section{Optimized Voxel-Based Morphometry Analysis}

The imaging data analysis was performed using Statistical Parametric Mapping version 8 software $^{1}$ implemented in MATLAB 7.4. As the basketball players were taller than novices and the brain volume varied with the height in individuals, we applied the optimized VBM approach (Good et al., 2001) to compare the gray matter volumes in two groups by creating a study-specific template. In the pre-processing step, each reoriented image was segmented (unified segmentation) into gray matter, white matter and cerebrospinal fluid (Ashburner and Friston, 2005). Segmented gray matter images of all subjects were rigidly transformed and averaged to create the studyspecific template. The aligned gray matter images in each subject were normalized with the study-specific template. Modulation

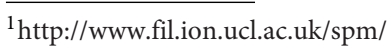


with Jacobian determinant was used to correct volume changes caused by spatial normalization. The modulated images were finally transformed into the Montreal Neurological Institute space and smoothed with a 6-mm full-width at half maximum Gaussian kernel.

Pre-processed gray matter images in basketball players and novices were compared with a two-sample $t$-test (second-level) run at the whole brain level. The height of subject was set as a covariate to exclude the potential contamination caused by different sizes of brain in two groups. The $t$-map was set at a corrected significance level of $p<0.05$. AlphaSim correction (REST_V1.8) with Monte Carlo simulation was used to correct for multiple comparisons (Poline et al., 1997; Song et al., 2011) with consideration both for the individual voxel probability and cluster size threshold. Based on the results of optimized VBM analysis, brain areas with larger gray matter volume in basketball players were identified (Figure 1). We also tested the correlation between gray matter volumes in these areas and training time in basketball players with the Pearson correlation coefficient. These areas were further selected as the seeds for the subsequent functional connectivity analyses. The seed was defined as a $6 \mathrm{~mm}$ radius sphere.

\section{Functional Connectivity Analysis}

We defined five seeds (Figure 1) for the resting-state functional imaging analysis. The pre-processing for the resting-state functional imaging analysis included slice time correction, rigid body movement correction, normalization of the functional images by directly registering onto the Montreal Neurological Institute echo planar imaging template (interpolated spatial resolution $3 \mathrm{~mm}^{3} \times 3 \mathrm{~mm}^{3} \times 3 \mathrm{~mm}^{3}$ ) and spatially smoothing ( $6 \mathrm{~mm}$ full-width at half maximum). The voxel-wise correlation analysis was conducted after the initial imaging data were temporally filtered $(0.01-0.08 \mathrm{~Hz})$. The resting-state time series of the five selected seed regions were extracted using MarsBaR toolbox (Brett et al., 2002). The correlation coefficient ( $r$-value) between the seed region ( $6 \mathrm{~mm}$ radius sphere) and other voxels of the whole brain (excluding those in the seed region) was computed. The correlation coefficient was converted into a $\mathrm{Z}$ score by Fisher's $r$-to- $z$ transformation to generate a contrast matrix for each seed region in each subject. For the group data analysis, we used a two-sample $t$-test to compare the difference between basketball players and novices (Figure 2). The $t$-test was repeated five times (one $t$-test for each seed region). AlphaSim correction with Monte Carlo simulation was used to correct for multiple comparisons. The $t$-map was set at a corrected significance level of $p<0.05$.

One purpose of our study was to investigate the differences in functional connectivity between two groups as they were applied to the cortical networks with potential interests. We predicted the differences in functional connectivity between two subject groups would be due to different motor and cognitive functions in them which were likely related to different cortical networks. We specifically focused on three cortical networks, including the default mode network, salience network and executive control network. Therefore, we illustrated the data with functional connectivity from five seed regions (superimposed results of $5 t$-tests) and projected the data onto a network brain template (Brain NetViewer ${ }^{2}$; Xia et al., 2013; Figure 3). The clusters of involved brain areas were extracted separately for each seed region and an ICBM152 brain template was used (REST_V1.8; Song et al., 2011). This three-dimensional volume-to-surface mapping provided more intuitive information about the seed regions and other functionally connected brain areas within three cortical networks and exhibited the spatial distribution of these cortical networks in the brain (Margulies et al., 2013).

\section{RESULTS}

\section{Gray Matter Volume}

We used an optimized VBM technique to analyze T1-weighted anatomical scanning and set height as a covariate to correct

$\overline{{ }^{2} \text { http://www.nitrc.org/projects/bnv/ }}$

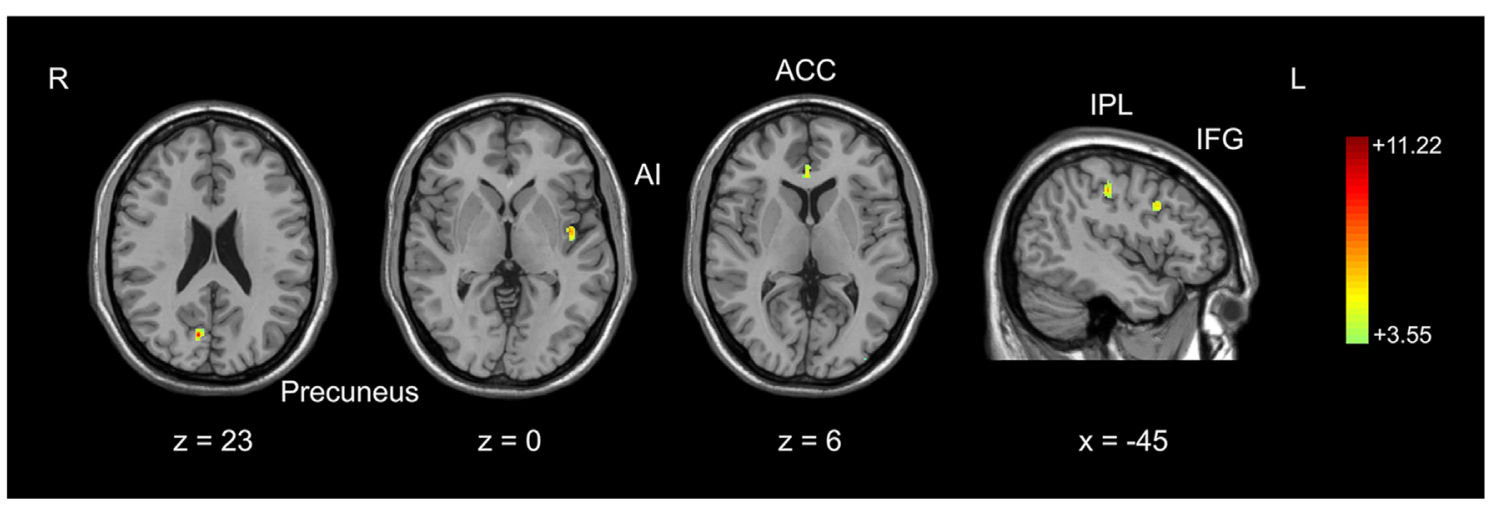

FIGURE 1 | Comparison of gray matter volume between basketball players and novices. Cortical areas with more gray matter volume in basketball players compared to novices was shown. Color scale represents the significant $t$-values (corrected $p<0.05$ ). $L=l$ left; $R=$ right. Abbreviations for brain areas: ACC, anterior cingulate cortex; Al, anterior insula; IFG, inferior frontal gyrus; IPL, inferior parietal lobule. 


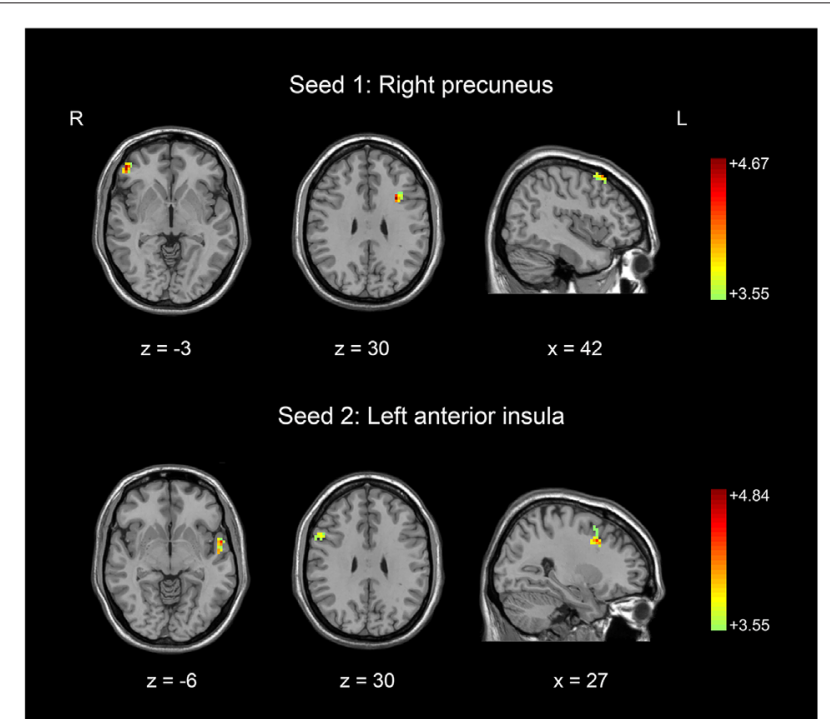

Seed 3: Right anterior cingulate cortex
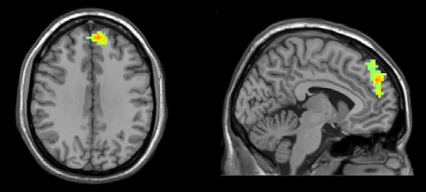

$x=-6$

$z=33$

Seed 4: Left inferior frontal gyrus
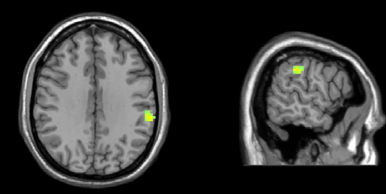

$x=-60$

$z=33$

Seed 5: Left inferior parietal lobule
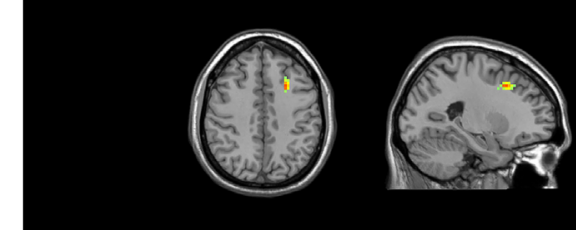

$x=-24$

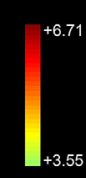

$z=42$

FIGURE 2 | Comparison of resting-state functional connectivity between basketball players and novices. Higher resting-state functional connectivity in basketball players compared to novices was shown. Five seed regions (three in the left brain and two in the right brain) were extracted. Color scale represents the significant $t$-values (corrected $p<0.05$ ).

for the potential effect of contamination caused by different brain sizes in two subject groups. It was found that basketball players had larger gray matter volumes than novices in multiple brain areas. These areas included right precuneus, left anterior insula (AI), right anterior cingulate cortex (ACC), left inferior frontal gyrus (IFG) and left inferior

parietal lobule (IPL; Figure 1; Table 1). These areas were selected as the seeds for the functional connectivity analyses. The inverse contrast analysis did not show larger volumes in novices compared to players in any brain areas. No significant correlation was found between the gray matter volume and training time in five seed regions in basketball players.

\section{Resting-State Functional Connectivity}

Five seed regions were connected with multiple brain areas at resting state both in basketball players and novices. Importantly, we found resting-state functional connectivity is different for basketball players and healthy controls in functional networks related to various brain areas (Figure 2; Table 2). Specifically, the right precuneus showed more resting connectivity with right inferior orbitofrontal gyrus (IOG), left pars opercularis (POP) of the IFG and right middle frontal gyrus (MFG) in the basketball players group. The connectivity between left AI and right MFG, right IFG, left superior temporal poles (STP) was stronger in the player group than that in the novice group. Right ACC was more functionally connected with left medial superior frontal gyrus (MSFG) in the basketball player group. Left IFG was more functionally connected with the left IPL while the left IPL was more functionally connected with the left MFG in the basketball player group compared to the novice group. On the other hand, the reversed comparison did not find stronger connectivity between seed region and any other cortical areas in novices compared to basketball players.

Furthermore, we projected the data onto a network brain template. It was confirmed that right precuneus had stronger connectivity with left POP of the IFG and right IOG, MFG in default mode network in basketball players compared to that in novices (Figure 3A). For the salience network (Figure 3B), left AI had stronger connectivity with left STP and right IFG while right ACC was more functionally connected with left MSFG in basketball players than novices. For the executive control network (Figure 3C), functional connectivity between left IFG and IPL and that between IPL and MFG was stronger in basketball players than that in novices.

\section{DISCUSSION}

We investigated the difference in brain structure and restingstate functional connectivity between basketball players and novices. The novel finding was that basketball players showed greater gray matter volume in five brain areas (Figure 1). Furthermore, basketball players displayed higher resting-state functional connectivity between these five seed regions and other cortical areas compared to novices. These cortical areas are located in default mode network, salience network and executive control network which are related to motor and cognitive functions in basketball players (Figures 2, 3).

\section{Gray Matter Volume}

We found larger volume of gray matter in right precuneus, left AI, right ACC, left IFG and left IPL in basketball players compared to novices. Precuneus is associated with processing 


\section{A Default Mode Network}

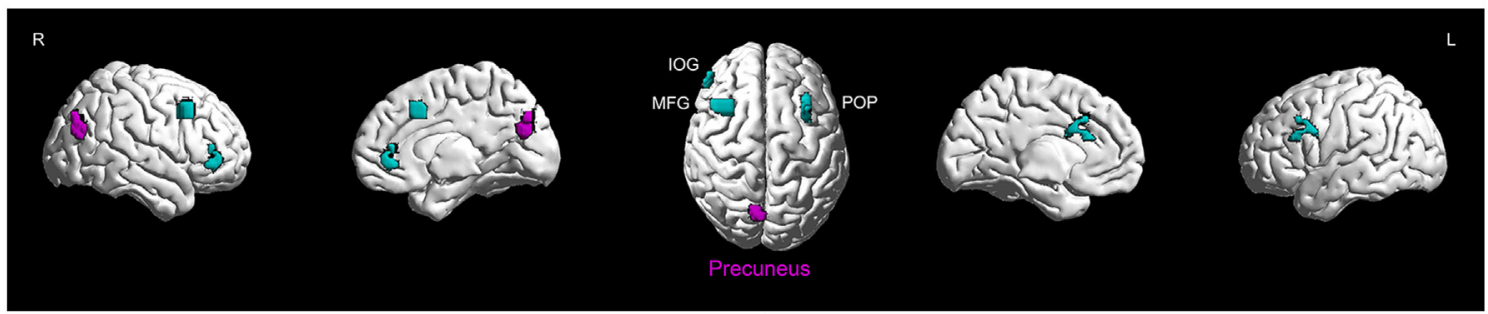

B Salience Network

C Executive Control network

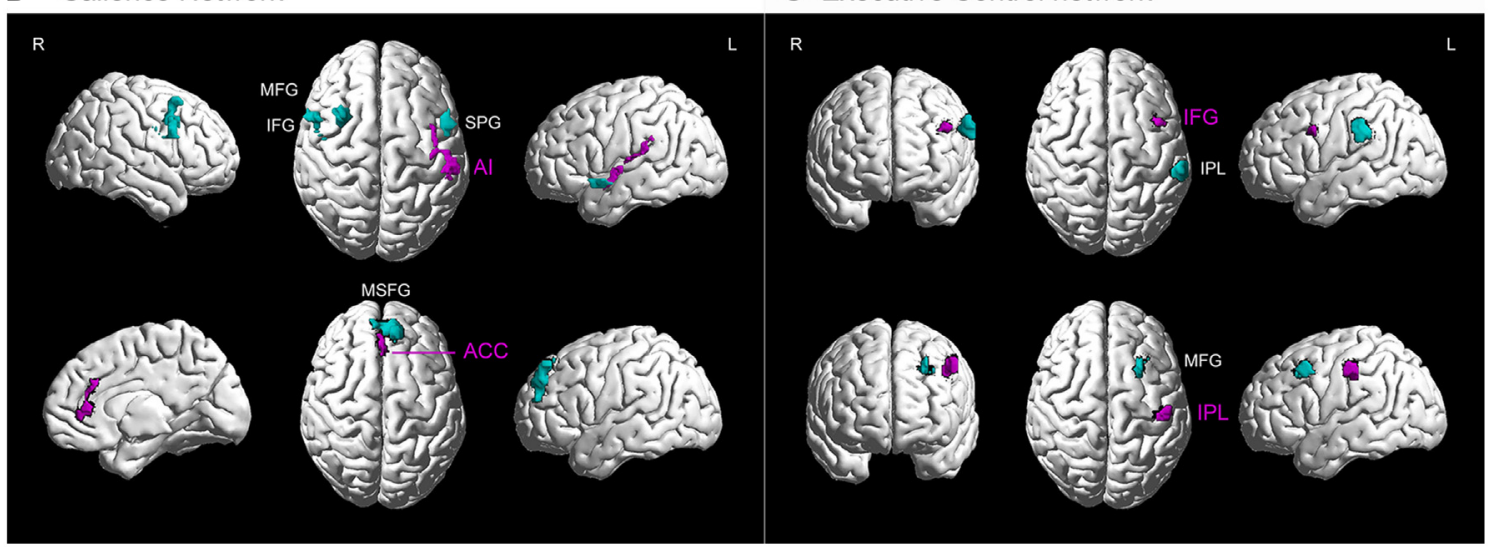

FIGURE 3 | Higher resting-state functional connectivity in basketball players related to three cortical networks. Five seed regions (purple) with enhanced gray matter volume showed stronger functional connectivity with several brain areas (green) in the basketball players compared to novices. These functionally connected brain areas are parts of default mode network (A), executive control network (B) and attention network $(\mathbf{C})$. $L=$ left; $R=$ right. Abbreviations for brain areas: ACC, anterior cingulate cortex; Al, anterior insula; IFG, inferior frontal gyrus; IOG, inferior orbitofrontal gyrus; IPL, inferior parietal lobule; MFG, middle frontal gyrus; MSFG, medial superior frontal gyrus; POP, pars opercularis (of the inferior frontal gyrus); STP, superior temporal pole.

spatial information during motor execution and preparation (Kawashima et al., 1995; Cavanna and Trimble, 2006). In particular, precuneus was involved in target tracking tasks with special requirement for attention to spatial information (Wenderoth et al., 2005; Cavanna and Trimble, 2006). Regular practicing skill of tracking the frequently moving targets in basketball may explain the volume increase in precuneus in basketball players compared to novices. Insula shows high activation during complex behavioral tasks (Craig, 2009) and plays an important role in making a rapid decision in a risky situation (Craig, 2002; Singer et al., 2009). Such ability with superior motor related perceptual functions is

TABLE 1 | Gray matter volumes in five seed areas in basketball players and novices (players $>$ novices).

\begin{tabular}{llrrrrr}
\hline Brain region & Side & $\boldsymbol{x}$ & $\boldsymbol{y}$ & $\boldsymbol{z}$ & Voxels & $\boldsymbol{t}$-value \\
\hline Precuneus (BA 31) & $\mathrm{R}$ & 11 & -74 & 23 & 52 & 8.52 \\
Anterior insula (BA 13) & $\mathrm{L}$ & -42 & -6 & -0 & 136 & 7.88 \\
Anterior cingulate & $\mathrm{R}$ & 2 & 32 & 6 & 35 & 6.32 \\
cortex (BA 32) & & & & & & \\
Inferior frontal gyrus (BA 9) & $\mathrm{L}$ & -45 & 11 & 33 & 48 & 6.75 \\
Inferior parietal lobule (BA 3) & $\mathrm{L}$ & -45 & -24 & 44 & 75 & 7.50
\end{tabular}

BA, Brodmann's area; $L$, left; $R$, right. Coordinates refer to Talairach space. Brain areas with corrected $p<0.05$ were listed. required in basketball because the players often perceive their self-positioning in the court and make decision in offending/defending strategy (Bar-Eli and Tractinsky, 2000; Llorca-Miralles et al., 2013; Kinrade et al., 2015). Our results also confirmed that physical exercise induces volume increase in the ACC (Flöel et al., 2010; Prakash et al., 2010) and supported the opinion that ACC is the major neuronal substrate for attention (Osaka et al., 2007) and action selection (Rushworth, 2008). The IFG is related to the action observation and imitation (Buccino et al., 2004; Calvo-Merino et al., 2005, 2006; Iseki et al., 2008; Caspers et al., 2010), which may be essential in basketball because the programming and execution of motor plan in basketball highly relies on the action observation of the opponent players (Fujii et al., 2014a,b). The IPL is important for complex cognitive functions, including visual perception, spatial perception and visuomotor integration (Anderson, 2011). The present finding of more gray matter volume in IPL in basketball players was consistent with previous evidence from neuroimaging studies that IPL was activated during action observation (Grèzes and Decety, 2001; Buccino et al., 2004; Hamilton and Grafton, 2006; Chong et al., 2008) and anticipation with correct understanding of the movement (Rizzolatti et al., 2006).

The basketball players in the present study are top athletes in China. The expertise in basketball skill largely varies 
TABLE 2 | Resting-state functional connectivity between seed regions and other brain areas in basketball players and novices (players > novices).

\begin{tabular}{|c|c|c|c|c|c|c|}
\hline Brain region & Side & $x$ & $y$ & $z$ & Voxels & $t$-value \\
\hline \multicolumn{7}{|l|}{ Seed 1: Right precuneus } \\
\hline Inferior orbitofrontal gyrus (BA 47) & $\mathrm{R}$ & 51 & 45 & -3 & 53 & 4.43 \\
\hline Inferior frontal gyrus (pars opercularis, BA 47) & $L$ & -30 & 12 & 30 & 58 & 4.52 \\
\hline Middle frontal gyrus (BA 8) & $\mathrm{R}$ & 42 & 18 & 57 & 49 & 3.89 \\
\hline \multicolumn{7}{|l|}{ Seed 2: Left anterior insula } \\
\hline Superior temporal pole (BA 38) & $\mathrm{L}$ & -57 & 3 & -6 & 34 & 4.05 \\
\hline Middle frontal gyrus (BA 6/BA 8) & $\mathrm{R}$ & 27 & 12 & 39 & 84 & 4.39 \\
\hline Inferior frontal gyrus (BA 9) & $\mathrm{R}$ & 54 & 12 & 30 & 48 & 3.82 \\
\hline \multicolumn{7}{|l|}{ Seed 3: Right anterior cingulate cortex } \\
\hline Medial superior frontal gyrus (BA 9) & $\mathrm{L}$ & -6 & 51 & 33 & 167 & 4.96 \\
\hline \multicolumn{7}{|l|}{ Seed 4: Left inferior frontal gyrus } \\
\hline Inferior parietal lobule (BA 40) & $\mathrm{L}$ & -60 & -33 & 33 & 31 & 4.15 \\
\hline \multicolumn{7}{|l|}{ Seed 5: Left inferior parietal lobule } \\
\hline Middle frontal gyrus (BA 8) & $\mathrm{L}$ & -24 & 15 & 42 & 25 & 3.78 \\
\hline
\end{tabular}

BA, Brodmann's area; L, left; R, right. Coordinates refer to Talairach space. Brain areas with corrected $p<0.05$ were listed.

depending on the special role that the athlete plays on the court (e.g., point guard and center have completely different playing styles) although our subjects have a similar duration in basketball training. The diversity in playing style with similar training duration might explain why we did not find significant correlation between gray matter volume and training duration in basketball players. This is different from our recent transcranial magnetic stimulation study in badminton players whose motor cortical excitation and inhibition are correlated to training years (Dai et al., 2016).

\section{Functional Connectivity in Cortical Neuronal Networks}

Independent component analysis and seed-based correlation analysis are two most common techniques used in functional connectivity data analysis (Biswal et al., 1995; Fox et al., 2005). Although network map obtained from the independent component analysis may be used as a reference to interpret the results from seed-based correlation analysis, two data analysis techniques are based on different mathematical models (Calhoun et al., 2001; van de Ven et al., 2004; Joel et al., 2011). Independent component analysis provides a means to test several spatially separated cortical networks at once. However, the value of a voxel being tested with the independent component analysis represents the correlation between the time series of this voxel and the mean time series of a particular cortical network. The interpretation for these data-driven networks largely depends on the predetermined number of components for production which changes the patterns of spatially separated cortical networks. The interpretation is further challenged by the complexity of noise identification process which is often determined with system selection by the user. On the other hand, seed-based correlation analysis requires the selection of the seed regions. Voxel value from seed-based correlation analysis reflects the degree to which the time series of a tested voxel is correlated with the time series of the seed region. Owing to inherent simplicity, high sensitivity and ease of interpretation, seed-based correlation analysis is widely used to test the functional connectivity between a given seed region and the other cortical areas. We defined five seed regions with larger gray matter volumes in basketball players through VBM analysis at the first step in our study. Therefore, we specifically tested whether the correlations of time series between the seed regions and other cortical areas were different in basketball players and novices. A seed-based correlation approach is likely better and more practicable to identify the difference between two groups in our study. In addition, it may be worth mentioning that previous studies reported similar results when same resting-state fMRI data set was processed by independent component analysis and seed-based correlation analysis techniques (Damoiseaux et al., 2006).

Default mode network includes precuneus, posterior cingulate, medial prefrontal cortex and inferior parietal cortex (Raichle et al., 2001; Fox et al., 2005). We found greater connectivity between precuneus and medial prefrontal cortex in basketball players, supporting the functions of precuneus and the medial prefrontal cortex as the core nodes in default mode network (Martinelli et al., 2013). Our results were consistent with previous study performed in musicians that long-term motor learning and expertise experience lead to resting-state functional connectivity changes in the default mode network (Fauvel et al., 2014). As precuneus and medial prefrontal cortex highly involve in self-related episodic memory (Dörfel et al., 2009), it may be explained that higher activity in the default mode network is caused by frequently processed self-related episodic memory in basketball playing.

Salience network is composed of AI, dorsal ACC (dACC) and ventrolateral prefrontal cortex (Seeley et al., 2007; Chan et al., 2008). We found high connectivity between AI, frontal cortex and STP in basketball players. The result may be consistent with the notion that AI is highly involved in extracting key salient stimuli from multiple inputs (Menon and Uddin, 2010). Our previous study also reported greater AI activity when basketball players noticed incorrect anticipation during observation of a basketball free throw (Wu et al., 2013). Frontal cortex is responsible for the episodic memory extraction 
(McDermott et al., 1999; Wagner, 1999; Lepage et al., 2000; Cabeza et al., 2002) while STP is related to the storage of semantic memory (Markowitsch, 1995; Simmons and Martin, 2009). Our results support the idea that memory extraction and storage are essential in basketball. We also found strong connectivity between ACC and MSFG (one part of frontal cortex) in basketball players. As ACC is related to the detection and processing of salient information and monitoring of errors (Kiehl et al., 2000; Hester et al., 2005; Etkin et al., 2011), our results may suggest that the process of semantic memory with interaction between ACC and frontal cortical area is important to maintain the high performance for the basketball players.

Executive control network is distributed in the frontoparietal system which comprises the dorsolateral prefrontal cortex and posterior parietal cortex. Particularly, frontal cortex is a primary region in modulating regular allocation of spatial attention (Schafer and Moore, 2011) and parietal cortex is heavily involved in spatial awareness (Behrmann et al., 2004). Our results that connectivity between the IFG, MFG and IPL is stronger in basketball players compared to novices may verify the idea that executive control network is the key structure for converting selective and spatial attention (Wu et al., 2007) in athletes with high motor expertise.

\section{Motor and Cognitive Functions in Basketball Players}

Basketball is a confrontational sport with open motor skill in which movements and actions of the players largely depend on the understanding of the environment and actions of other players (both the team mates and the players on the opposite side; Schmidt and Wrisberg, 2008). Our results with larger gray matter volume and higher functional connectivity in basketball players involved in multiple cortical areas and various cortical networks suggest that complex motor and cognitive functions combining visual search, perceptual anticipation and action execution are required in the development of motor expertise (Abernethy, 1996; Vickers, 2004). It is not surprising that the gray matter volumes in motor related cortical areas increase and show enhanced connectivity with other cortical areas as frequent engagement of these cortical areas during long-term training induces the cortical plasticity in the underlying neuronal components and facilitates the communication of these components within the networks (Fries, 2005; Lewis et al., 2009; Duan et al., 2012). Interestingly, we found gray matter volume increases and functional connectivity enhanced in a wide range of cortical areas among three different functional networks in basketball players. Structural and functional changes in these areas largely contribute to the improvement of cognitive functions including temporal and spatial attention (Wright et al., 2013; Wu et al., 2013), memory processing (Wan et al., 2011; Wang et al., 2013), decision making and error correction (Koelewijn et al., 2008; Cocchi et al., 2013) in the population with professional experience. Our results are consistent with previous studies performed in other sports players (Di et al., 2012; Wang et al., 2013) and support the view that development of motor expertise relies on the improvement both in motor and cognitive functions (Aglioti et al., 2008). Future studies with further consideration about the interaction and mutual advantage between motor and cognitive components may help elucidate the mechanisms of cortical plasticity during the acquisition of high-level motor expertise.

We did not find increase in gray matter volume and functional connectivity related to primary motor cortex in basketball players compared to novices. This is consistent with previous studies in cohorts with other motor expertise, such as musicians (Fauvel et al., 2014), taxi drivers (Maguire et al., 2000) and athletes (Wei et al., 2011; Di et al., 2012; Wang et al., 2013). Interestingly, our previous studies with transcranial magnetic stimulation found increased motor cortical excitability during different motor tasks in athletes (Wang et al., 2014; Dai et al., 2016). It may be inferred that changes (both gray matter volume and functional connectivity) in other motor and cognitive related brain areas alter the cortico-cortical projections to the primary motor cortex and eventually lead to the increased output from the motor cortex in athletes. However, we cannot exclude the possibility that functional or even structural changes occur in the primary motor cortex itself after different courses of long-term motor training (Gaser and Schlaug, 2003; Draganski et al., 2004).

\section{Limitations}

We investigated the morphological and functional differences between athletes and novices with a cross-sectional paradigm. It may be argued that larger gray matter volume and stronger functional connectivity observed in basketball players are not induced by long-term training but simply due to the natural property in this cohort which potentially leads to an "expert" brain with better structure and functions during development. Although our optimized VBM analysis partly ruled out the effect caused by inherent brain size difference in two groups, the question how cortical plasticity with long-term training is related to the structural and functional changes in the brain should be further addressed by longitudinal studies performed along the whole career of the athletes.

In addition, stronger connectivity in three functional networks was identified in the basketball players. It has long been controversial whether and how the resting-state functional connectivity represents the anatomical and biological connections in the brain (Raichle et al., 2001; Fox et al., 2005, 2006). Our study does not directly approach the question how the strong functional connectivity in the basketball players is related to the biological changes after the long-term training. However, our findings that functional connectivity was relevant to the seed region where structural (gray matter volume) difference was found between two groups and that we did not perform any pre-selection in determination of the seed region might suggest the potential biological changes with cortical plasticity after long-term training in athletes. The opinion is also consistent with the evidence obtained from neuroimaging studies performed in musicians that changes in functional map are often accompanied with 
structural changes during acquisition of motor expertise (Schlaug, 2001).

A reversed question is whether the result of functional connectivity analysis is affected by the difference in seed regions determined in the VBM approach. The findings that higher resting-state functional connectivity seen in basketball players compared to novices in the present study was based on the whole brain wide correlation analysis and that the cortical areas where higher functional connectivity were found (except for the seed regions) did not show greater gray matter volumes might partly deny the cause of increase in functional connectivity with simple changes in seed sizes. Similar increases both in gray matter volumes in seed regions and functional connectivity with the seed regions were also found in musicians (Fauvel et al., 2014). In addition, the reference time series in two subject groups are likely slightly different due to more gray matter volume in basketball players compared to novices when parametric approach is used in our time domain analysis (Friston et al., 1994). The comparison for functional connectivity between two groups may further be confounded by the partial volume effect around the seed regions (Müller-Gärtner et al., 1992). Although we performed additional masks in the seed regions by excluding voxels with low gray matter density (value below 0.3 ) to minimize partial volume effect, it may still be argued that the functional connectivity in novices is potentially underestimated with the fact that the selected seeds in the novices are contaminated by gray matter in other adjacent cortical areas or even white matter. The interaction between structural and functional changes during the long-term course of motor training is complex and the answer to this complex question again requires future work with longitudinal studies performed in the training course.

\section{REFERENCES}

Abernethy, B. (1996). Training the visual-perceptual skills of athletes. Insights from the study of motor expertise. Am. J. Sports Med. 24,S89-S92.

Aglioti, S. M., Cesari, P., Romani, M., and Urgesi, C. (2008). Action anticipation and motor resonance in elite basketball players. Nat. Neurosci. 11, 1109-1116. doi: 10.1038/nn.2182

Alvarez, J. A., and Emory, E. (2006). Executive function and the frontal lobes: a meta-analytic review. Neuropsychol. Rev. 16, 17-42. doi: 10.1007/s11065-0069002-x

Anderson, R. A. (2011). "Inferior parietal lobule function in spatial perception and visuomotor integration," in Comprehensive Physiology, ed. D. M. Pollock 483-518.

Ashburner, J., and Friston, K. J. (2000). Voxel-based morphometry-the methods. Neuroimage 11, 805-821. doi: 10.1006/nimg.2000.0582

Ashburner, J., and Friston, K. J. (2005). Unified segmentation. Neuroimage 26, 839-851. doi: 10.1016/j.neuroimage.2005.02.018

Bar-Eli, M., and Tractinsky, N. (2000). Criticality of game situations and decision making in basketball: an application of performance crisis perspective. Psychol. Sport Exerc. 1, 27-39. doi: 10.1016/s1469-0292(00) 00005-4

Behrmann, M., Geng, J. J., and Shomstein, S. (2004). Parietal cortex and attention. Curr. Opin. Neurobiol. 14, 212-217. doi: 10.1016/j.conb.2004.03.012

Biswal, B., Yetkin, F. Z., Haughton, V. M., and Hyde, J. S. (1995). Functional connectivity in the motor cortex of resting human brain using echo-planar MRI. Magn. Reson. Med. 34, 537-541. doi: 10.1002/mrm.1910340409

\section{CONCLUSION}

Using structural and resting-state functional imaging techniques, the present study revealed larger volumes of gray matter in five seed regions and higher functional connectivity in default mode network, salience network and executive control network in basketball players compared to novices. We conclude that the morphology and functional connectivity in cortical neuronal networks in athletes and novices are different, and the differences may be related to higher level of motor expertise in athletes with better motor and cognitive functions.

\section{AUTHOR CONTRIBUTIONS}

X-YT and Y-LP conceived, designed and conducted experiments. YW analyzed data, interpreted results and wrote the manuscript. X-PL, L-LZ, WD and HZ helped conduct experiments and edited the manuscript. ZN, JW and JZ revised the manuscript. All authors approved the submitted version.

\section{FUNDING}

The present study was funded by the National Natural Science Foundation of China (No. 31470051, No. 31371056), Shanghai Pudong New Area Health Bureau (No. PWZxkq 2011-02) and Shanghai Key Lab of Human Performance (Shanghai University of Sport, No. 11DZ2261100).

\section{ACKNOWLEDGMENTS}

We thank collaborators in East China Normal University for data acquisition.

Blakemore, S. J., and Frith, U. (2005). The learning brain: lessons for education: a précis. Dev. Sci. 8, 459-465. doi: 10.1111/j.1467-7687.2005. 00434.x

Bressler, S. L., and Menon, V. (2010). Large-scale brain networks in cognition: emerging methods and principles. Trends Cogn. Sci. 14, 277-290. doi: 10.1016/j. tics.2010.04.004

Brett, M. A. J., Anton, J. L., Valabregue, R., and Poline, J. B. (2002). Region of interest analysis using an spm toolbox. Neuroimage 13, 210-217.

Buccino, G., Lui, F., Canessa, N., Patteri, I., Lagravinese, G., Benuzzi, F., et al. (2004). Neural circuits involved in the recognition of actions performed by nonconspecifics: an fMRI study. J. Cogn. Neurosci. 16, 114-126. doi: 10. 1162/089892904322755601

Buckner, R. L., Andrews-Hanna, J. R., and Schacter, D. L. (2008). The brain's default network: anatomy, function and relevance to disease. Ann. N Y Acad. Sci. 1124, 1-38. doi: 10.1196/annals.1440.011

Cabeza, R., Dolcos, F., Graham, R., and Nyberg, L. (2002). Similarities and differences in the neural correlates of episodic memory retrieval and working memory. Neuroimage 16, 317-330. doi: 10.1006/nimg.2002.1063

Calhoun, V. D., Adali, T., Pearlson, G. D., and Pekar, J. J. (2001). A method for making group inferences from functional MRI data using independent component analysis. Hum. Brain Mapp. 14, 140-151. doi: 10.1002/ hbm.1048

Calvo-Merino, B., Glaser, D. E., Grèzes, J., Passingham, R. E., and Haggard, P. (2005). Action observation and acquired motor skills: an fMRI study with expert dancers. Cereb. Cortex 15, 1243-1249. doi: 10.1093/cercor/ bhi007 
Calvo-Merino, B., Grèzes, J., Glaser, D. E., Passingham, R. E., and Haggard, P. (2006). Seeing or doing? Influence of visual and motor familiarity in action observation. Curr. Biol. 16, 1905-1910. doi: 10.1016/j.cub.2006.07.065

Caspers, S., Zilles, K., Laird, A. R., and Eickhoff, S. B. (2010). ALE meta-analysis of action observation and imitation in the human brain. Neuroimage 50, 1148-1167. doi: 10.1016/j.neuroimage.2009.12.112

Cavanna, A. E., and Trimble, M. R. (2006). The precuneus: a review of its functional anatomy and behavioural correlates. Brain 129, 564-583. doi: 10. 1093/brain/awl004

Chan, R. C., Shum, D., Toulopoulou, T., and Chen, E. Y. H. (2008). Assessment of executive functions: review of instruments and identification of critical issues. Arch. Clin. Neuropsychol. 23, 201-216. doi: 10.1016/j.acn.2007.08.010

Chong, T. T., Williams, M. A., Cunnington, R., and Mattingley, J. B. (2008). Selective attention modulates inferior frontal gyrus activity during action observation. Neuroimage 40, 298-307. doi: 10.1016/j.neuroimage.2007.11.030

Cocchi, L., Halford, G. S., Zalesky, A., Harding, I. H., Ramm, B. J., Cutmore, T., et al. (2014). Complexity in relational processing predicts changes in functional brain network dynamics. Cereb. Cortex 24, 2283-2296. doi: 10. 1093/cercor/bht075

Cocchi, L., Zalesky, A., Fornito, A., and Mattingley, J. B. (2013). Dynamic cooperation and competition between brain systems during cognitive control. Trends Cogn. Sci. 17, 493-501. doi: 10.1016/j.tics.2013.08.006

Craig, A. D. (2002). How do you feel? Interoception: the sense of the physiological condition of the body. Nat. Rev. Neurosci. 3, 655-666. doi: 10.1038/nrn894

Craig, A. D. (2009). How do you feel-now? The anterior insula and human awareness. Nat. Rev. Neurosci. 10, 59-70. doi: 10.1038/nrn2555

Dai, W., Pi, Y., Ni, Z., Tan, X., Zhang, J., and Wu, Y. (2016). Maintenance of balance between motor cortical excitability and inhibition after long-term training. Neuroscience 336, 114-122. doi: 10.1016/j.neuroscience. 2016.08.053

Damoiseaux, J. S., Rombouts, S. A., Barkhof, F., Scheltens, P., Stam, C. J., Smith, S. M., et al. (2006). Consistent resting-state networks across healthy subjects. Proc. Natl. Acad. Sci. U S A 103, 13848-13853. doi: 10.1073/pnas. 0601417103

Di, X., Zhu, S., Jin, H., Wang, P., Ye, Z., Zhou, K., et al. (2012). Altered resting brain function and structure in professional badminton players. Brain Connect. 2, 225-233. doi: 10.1089/brain.2011.0050

di Pellegrino, G., Fadiga, L., Fogassi, L., Gallese, V., and Rizzolatti, G. (1992). Understanding motor events: a neurophysiological study. Exp. Brain Res. 91, 176-180. doi: 10.1007/bf00230027

Dörfel, D., Werner, A., Schaefer, M., von Kummer, R., and Karl, A. (2009). Distinct brain networks in recognition memory share a defined region in the precuneus. Eur. J. Neurosci. 30, 1947-1959. doi: 10.1111/j.1460-9568.2009.06973.x

Dosenbach, N. U., Fair, D. A., Cohen, A. L., Schlaggar, B. L., and Petersen, S. E. (2008). A dual-networks architecture of top-down control. Trends Cogn. Sci. 12, 99-105. doi: 10.1016/j.tics.2008.01.001

Draganski, B., Gaser, C., Busch, V., Schuierer, G., Bogdahn, U., and May, A. (2004). Neuroplasticity: changes in grey matter induced by training. Nature 427, 311-312. doi: 10.1038/427311a

Duan, X., He, S., Liao, W., Liang, D., Qiu, L., Wei, L., et al. (2012). Reduced caudate volume and enhanced striatal-DMN integration in chess experts. Neuroimage 60, 1280-1286. doi: 10.1016/j.neuroimage.2012.01.047

Eckert, M. A., Menon, V., Walczak, A., Ahlstrom, J., Denslow, S., Horwitz, A., et al. (2009). At the heart of the ventral attention system: the right anterior insula. Hum. Brain Mapp. 30, 2530-2541. doi: 10.1002/hbm.20688

Etkin, A., Egner, T., and Kalisch, R. (2011). Emotional processing in anterior cingulate and medial prefrontal cortex. Trends Cogn. Sci. 15, 85-93. doi: 10. 1016/j.tics.2010.11.004

Fauvel, B., Groussard, M., Chételat, G., Fouquet, M., Landeau, B., Eustache, F., et al. (2014). Morphological brain plasticity induced by musical expertise is accompanied by modulation of functional connectivity at rest. Neuroimage 90 , 179-188. doi: 10.1016/j.neuroimage.2013.12.065

Flöel, A., Ruscheweyh, R., Krüger, K., Willemer, C., Winter, B., Völker, K., et al. (2010). Physical activity and memory functions: Are neurotrophins and cerebral gray matter volume the missing link? Neuroimage 49, 2756-2763. doi: 10.1016/j.neuroimage.2009.10.043

Fox, M. D., Corbetta, M., Snyder, A. Z., Vincent, J. L., and Raichle, M. E. (2006). Spontaneous neuronal activity distinguishes human dorsal and ventral attention systems. Proc. Natl. Acad. Sci. U S A 103, 10046-10051. doi: 10 1073/pnas.0604187103

Fox, M. D., Snyder, A. Z., Vincent, J. L., Corbetta, M., Van Essen, D. C., and Raichle, M. E. (2005). The human brain is intrinsically organized into dynamic, anticorrelated functional networks. Proc. Natl. Acad. Sci. US A 102, 9673-9678. doi: 10.1073/pnas.0504136102

Fries, P. (2005). A mechanism for cognitive dynamics: neuronal communication through neuronal coherence. Trends Cogn. Sci. 9, 474-480. doi: 10.1016/j.tics. 2005.08.011

Friston, K. J., Worsley, K. J, Frackowiak, R. S., Mazziotta, J. C., and Evans, A. C (1994). Assessing the significance of focal activations using their spatial extent. Hum. Brain Mapp. 1, 210-220. doi: 10.1002/hbm.4600 10306

Fujii, K., Shinya, M., Yamashita, D., Kouzaki, M., and Oda, S. (2014a). Anticipation by basketball defenders: an explanation based on the threedimensional inverted pendulum model. Eur. J. Sport Sci. 14, 538-546. doi: 10. 1080/17461391.2013.876104

Fujii, K., Shinya, M., Yamashita, D., Oda, S., and Kouzaki, M. (2014b). Superior reaction to changing directions for skilled basketball defenders but not linked with specialised anticipation. Eur. J. Sport Sci. 14, 209-216. doi: 10 1080/17461391.2013.780098

Gallese, V., Fadiga, L., Fogassi, L., and Rizzolatti, G. (1996). Action recognition in the premotor cortex. Brain 119, 593-609. doi: 10.1093/brain/ 119.2 .593

Gaser, C., and Schlaug, G. (2003). Brain structures differ between musicians and non-musicians. J. Neurosci. 23, 9240-9245.

Good, C. D., Johnsrude, I. S., Ashburner, J., Henson, R. N., Friston, K. J., and Frackowiak, R. S. (2001). A voxel-based morphometric study of ageing in 465 normal adult human brains. Neuroimage 14, 21-36. doi: 10.1006/nimg. 2001.0786

Grèzes, J., and Decety, J. (2001). Functional anatomy of execution, mental simulation, observation and verb generation of actions: a meta-analysis. Hum. Brain Mapp. 12, 1-19. doi: 10.1002/1097-0193(200101)12:1<1::AIDHBM10>3.0.CO;2-V

Hamilton, A. F., and Grafton, S. T. (2006). Goal representation in human anterior intraparietal sulcus. J. Neurosci. 26, 1133-1137. doi: 10.1523/JNEUROSCI. 4551-05.2006

Hester, R., Foxe, J. J., Molholm, S., Shpaner, M., and Garavan, H. (2005). Neural mechanisms involved in error processing: a comparison of errors made with and without awareness. Neuroimage 27, 602-608. doi: 10.1016/j.neuroimage. 2005.04.035

Imfeld, A., Oechslin, M. S., Meyer, M., Loenneker, T., and Jancke, L. (2009). White matter plasticity in the corticospinal tract of musicians: a diffusion tensor imaging study. Neuroimage 46, 600-607. doi: 10.1016/j.neuroimage.2009. 02.025

Iseki, K., Hanakawa, T., Shinozaki, J., Nankaku, M., and Fukuyama, H. (2008). Neural mechanisms involved in mental imagery and observation of gait. Neuroimage 41, 1021-1031. doi: 10.1016/j.neuroimage.2008. 03.010

Jäncke, L., Koeneke, S., Hoppe, A., Rominger, C., and Hanggi, J. (2009). The architecture of the golfer's brain. PLoS One 4:e4785. doi: 10.1371/journal.pone. 0004785

Joel, S. E., Caffo, B. S., van Zijl, P. C., and Pekar, J. J. (2011). On the relationship between seed-based and ICA-based measures of functional connectivity. Magn. Reson. Med. 66, 644-657. doi: 10.1002/mrm.22818

Kawashima, R., Roland, P. E., and O'Sullivan, B. T. (1995). Functional anatomy of reaching and visuomotor learning: a positron emission tomography study. Cereb. Cortex 5, 111-122. doi: 10.1093/cercor/5.2.111

Kiehl, K. A., Liddle, P. F., and Hopfinger, J. B. (2000). Error processing and the rostral anterior cingulate: an event-related fMRI study. Psychophysiology 37, 216-223. doi: 10.1111/1469-8986.3720216

Kinrade, N. P., Jackson, R. C., and Ashford, K. J. (2015). Reinvestment, task complexity and decision making under pressure in basketball. Psychol. Sport Exerc. 20, 11-19. doi: 10.1016/j.psychsport.2015.03.007

Koelewijn, T., van Schie, H. T., Bekkering, H., Oostenveld, R., and Jensen, O. (2008). Motor-cortical beta oscillations are modulated by correctness of observed action. Neuroimage 40, 767-775. doi: 10.1016/j.neuroimage.2007. 12.018 
Lepage, M., Ghaffar, O., Nyberg, L., and Tulving, E. (2000). Prefrontal cortex and episodic memory retrieval mode. Proc. Natl. Acad. Sci. U S A 97, 506-511. doi: 10.1073/pnas.97.1.506

Lewis, C. M., Baldassarre, A., Committeri, G., Romani, G. L., and Corbetta, M. (2009). Learning sculpts the spontaneous activity of the resting human brain. Proc. Natl. Acad. Sci. U S A 106, 17558-17563. doi: 10.1073/pnas.09024 55106

Lisberger, S. G. (1988). The neural basis for learning of simple motor skills. Science 242, 728-735. doi: 10.1126/science.3055293

Llorca-Miralles, J., Sanchez-Delgado, G., Pinar, M. I., Cardenas, D., and Perales, J. C. (2013). Basketball training influences shot selection assessment: a multi-attribute decision-making approach. Rev. Psicol. Deporte 22, 223-226.

Maguire, E. A., Gadian, D. G., Johnsrude, I. S., Good, C. D., Ashburner, J., Frackowiak, R. S., et al. (2000). Navigation-related structural change in the hippocampi of taxi drivers. Proc. Natl. Acad. Sci. U S A 97, 4398-4403. doi: 10. 1073/pnas.070039597

Mantini, D., and Vanduffel, W. (2013). Emerging roles of the brain's default network. Neuroscientist 19, 76-87. doi: 10.1177/1073858412446202

Margulies, D. S., Böttger, J., Watanabe, A., and Gorgolewski, K. J. (2013). Visualizing the human connectome. Neuroimage 80, 445-461. doi: 10.1016/j. neuroimage.2013.04.111

Markowitsch, H. J. (1995). Which brain regions are critically involved in the retrieval of old episodic memory? Brain Res. Rev. 21, 117-127. doi: 10. 1016/0165-0173(95)00007-0

Martinelli, P., Sperduti, M., and Piolino, P. (2013). Neural substrates of the self-memory system: new insights from a meta-analysis. Hum. Brain Mapp. 34, 1515-1529. doi: 10.1002/hbm.22008

McDermott, K. B., Buckner, R. L., Petersen, S. E., Kelley, W. M., and Sanders, A. L. (1999). Set- and code-specific activation in frontal cortex: an fMRI study of encoding and retrieval of faces and words. J. Cogn. Neurosci. 11, 631-640. doi: 10.1162/089892999563698

McRobert, A. P., Williams, A. M., Ward, P., Eccles, D. W., and Ericsson, K. A. (2007). Contextual information and anticipation skill in cricket batting. J. Sport Exerc. Psychol. 29:S187.

Menon, V., and Uddin, L. Q. (2010). Saliency, switching, attention and control: a network model of insula function. Brain Struct. Funct. 214, 655-667. doi: 10. 1007/s00429-010-0262-0

Müller-Gärtner, H. W., Links, J. M., Prince, J. L., Bryan, R. N., McVeigh, E. and Leal, J. P. (1992). Measurement of radiotracer concentration in brain gray matter using positron emission tomography: MRI-based correction for partial volume effects. J. Cereb. Blood Flow Metab. 12, 571-583. doi: 10. 1038/jcbfm.1992.81

Osaka, M., Komori, M., Morishita, M., and Osaka, N. (2007). Neural bases of focusing attention in working memory: an fMRI study based on group differences. Cogn. Affect. Behav. Neurosci. 7, 130-139. doi: 10.3758/cabn.7. 2.130

Pascual-Leone, A., Amedi, A., Fregni, F., and Merabet, L. B. (2005). The plastic human brain cortex. Annu. Rev. Neurosci. 28, 377-401. doi: 10.1146/annurev. neuro.27.070203.144216

Poline, J. B., Worsley, K. J., Evans, A. C., and Friston, K. J. (1997). Combining spatial extent and peak intensity to test for activations in functional imaging. Neuroimage 5, 83-96. doi: 10.1006/nimg.1996.0248

Prakash, R. S., Snook, E. M., Motl, R. W., and Kramer, A. F. (2010). Aerobic fitness is associated with gray matter volume and white matter integrity in multiple sclerosis. Brain Res. 1341, 41-51. doi: 10.1016/j.brainres.2009. 06.063

Raichle, M. E., MacLeod, A. M., Snyder, A. Z., Powers, W. J., Gusnard, D. A., and Shulman, G. L. (2001). A default mode of brain function. Proc. Natl. Acad. Sci. U S A 98, 676-682. doi: 10.1073/pnas.98.2.676

Rizzolatti, G., Ferrari, P. F., Rozzi, S., and Fogassi, L. (2006). The inferior parietal lobule: where action becomes perception. Novartis Found. Symp. 270, 129-140; discussion 140-145, 164-169. doi: 10.1002/9780470034989. ch11

Rushworth, M. F. (2008). Intention, choice and the medial frontal cortex. Ann. N Y Acad. Sci. 1124, 181-207. doi: 10.1196/annals.1440.014

Schafer, R. J., and Moore, T. (2011). Selective attention from voluntary control of neurons in prefrontal cortex. Science 332, 1568-1571. doi: 10.1126/science. 1199892
Schlaug, G. (2001). The brain of musicians. a model for functional and structural adaptation. Ann. N Y Acad. Sci. 930, 281-299. doi: 10.1111/j.1749-6632.2001. tb05739.x

Schmidt, R. A., and Wrisberg, C. A. (2008). Motor Learning and Performance (Fourth Edn.) Champaign, IL: Human Kinetics.

Seeley, W. W., Menon, V., Schatzberg, A. F., Keller, J., Glover, G. H., Kenna, H., et al. (2007). Dissociable intrinsic connectivity networks for salience processing and executive control. J. Neurosci. 27, 2349-2356. doi: 10.1523/JNEUROSCI. 5587-06.2007

Simmons, W. K., and Martin, A. (2009). The anterior temporal lobes and the functional architecture of semantic memory. J. Int. Neuropsychol. Soc. 15, 645-649. doi: 10.1017/s1355617709990348

Singer, T., Critchley, H. D., and Preuschoff, K. (2009). A common role of insula in feelings, empathy and uncertainty. Trends Cogn. Sci. 13, 334-340. doi: 10. 1016/j.tics.2009.05.001

Song, X. W., Dong, Z. Y., Long, X. Y., Li, S. F., Zuo, X. N., Zhu, C. Z., et al. (2011). REST: a toolkit for resting-state functional magnetic resonance imaging data processing. PLoS One 6:e25031. doi: 10.1371/journal.pone.0025031

van de Ven, V. G., Formisano, E., Prvulovic, D., Roeder, C. H., and Linden, D. E. (2004). Functional connectivity as revealed by spatial independent component analysis of fmri measurements during rest. Hum. Brain Mapp. 22, 165-178. doi: $10.1002 / \mathrm{hbm} .20022$

Vickers, J. N. (2004). Cognitive control in motor expertise. Int. J. Sport Exerc. Psychol. 2, 274-300. doi: 10.1080/1612197x.2004.9671746

Wagner, A. D. (1999). Working memory contributions to human learning and remembering. Neuron 22, 19-22. doi: 10.1016/s0896-6273(00)80674-1

Wan, X., Nakatani, H., Ueno, K., Asamizuya, T., Cheng, K., and Tanaka, K. (2011). The neural basis of intuitive best next-move generation in board game experts. Science 331, 341-346. doi: 10.1126/science.1194732

Wang, B., Fan, Y., Lu, M., Li, S., Song, Z., Peng, X., et al. (2013). Brain anatomical networks in world class gymnasts: a DTI tractography study. Neuroimage 65, 476-487. doi: 10.1016/j.neuroimage.2012.10.007

Wang, Z., Wang, S., Shi, F. Y., Guan, Y., Wu, Y., Zhang, L. L., et al. (2014). The effect of motor imagery with specific implement in expert badminton player. Neuroscience 275, 102-112. doi: 10.1016/j.neuroscience.2014.06.004

Wei, G., Zhang, Y., Jiang, T., and Luo, J. (2011). Increased cortical thickness in sports experts: a comparison of diving players with the controls. PLoS One 6:e17112. doi: 10.1371/journal.pone.0017112

Wenderoth, N., Debaere, F., Sunaert, S., and Swinnen, S. P. (2005). The role of anterior cingulate cortex and precuneus in the coordination of motor behaviour. Eur. J. Neurosci. 22, 235-246. doi: 10.1111/j.1460-9568.2005.04176.x

Williams, A. M., Davids, K., and Williams, J. G. (1999). Visual Perception and Action in Sport. London: E and FN Spon.

Wright, M. J., Bishop, D. T., Jackson, R. C., and Abernethy, B. (2013). Brain regions concerned with the identification of deceptive soccer moves by higher-skilled and lower-skilled players. Front. Hum. Neurosci. 7:851. doi: 10.3389/fnhum. 2013.00851

Wu, C. T., Weissman, D. H., Roberts, K. C., and Woldorff, M. G. (2007). The neural circuitry underlying the executive control of auditory spatial attention. Brain Res. 1134, 187-198. doi: 10.1016/j.brainres.2006.11.088

Wu, Y., Zeng, Y., Zhang, L., Wang, S., Wang, D., Tan, X., et al. (2013). The role of visual perception in action anticipation in basketball athletes. Neuroscience 237, 29-41. doi: 10.1016/j.neuroscience.2013.01.048

Xia, M., Wang, J., and He, Y. (2013). BrainNet Viewer: a network visualization tool for human brain connectomics. PLoS One 8:e68910. doi: 10.1371/journal.pone. 0068910

Conflict of Interest Statement: The authors declare that the research was conducted in the absence of any commercial or financial relationships that could be construed as a potential conflict of interest.

Copyright (C) 2017 Tan, Pi, Wang, Li, Zhang, Dai, Zhu, Ni, Zhang and Wu. This is an open-access article distributed under the terms of the Creative Commons Attribution License (CC BY). The use, distribution and reproduction in other forums is permitted, provided the original author(s) or licensor are credited and that the original publication in this journal is cited, in accordance with accepted academic practice. No use, distribution or reproduction is permitted which does not comply with these terms. 